\title{
A multi-criteria decision method for sustainability assessment of the use phase of machine tool systems
}

\author{
Oliver Avram • Ian Stroud • Paul Xirouchakis
}

Received: 25 March 2010/Accepted: 28 July 2010/Published online: 13 August 2010

(C) Springer-Verlag London Limited 2010

\begin{abstract}
In recent years, going green has become a strategic priority in manufacturing which has evolved from the growing awareness of the need for environmentally friendly processes and products. Recent trends in developing new machining strategies able to support environmental protection and prevention of pollution in balance with socioeconomic needs and technical requirements inevitably require significant efforts in fundamental understanding of the actual energy and material flows needed to meet the machining requirements. This paper describes the methodology and software, Global Reasoning for Eco-Evaluation of Machining, developed to evaluate the use phase of a machine tool system with respect to a consistent set of technical, economical, and environmental criteria, as a part of the NEXT European project. The evaluation, based on the analytic hierarchy process, is conducted at two levels: (a) the process/part level which considers the local cutting environment along with the "actors" that are directly involved in the cutting area, their relationships, and the phenomena that occur from their interactions and (b) the system level which gathers together the main specifications of the machine tool system and the energy requirements associated with various activities performed in order to support the material removal processes. Two database structures were defined in order to store the entire amount of data needed to perform the analysis at each level. A detailed example of the evaluation carried out at the first level demonstrated the superior performance of the dry and
\end{abstract}

\footnotetext{
O. Avram $(\bowtie) \cdot$ I. Stroud $\cdot$ P. Xirouchakis

Institute of Mechanical Engineering, Swiss Federal Institute of

Technology Lausanne, STI-IGM-LICP,

Station 9,

1015 Lausanne, Switzerland

e-mail: oliver.avram@epfl.ch
}

minimum quantity lubrication alternatives over their wet machining counterpart when cutting power, cutting fluid consumption, and the machining time were considered as criteria for the performance evaluation of various milling tests.

Keywords AHP - Machine tool - Environmental impact . Machining alternatives

\section{Introduction}

Metal cutting processes are industrial processes in which metal parts are shaped by material removal processes involving the interaction between three main elements: the cutting tool, the material to be cut, and the cutting fluid. Application of the cutting fluids can be considered as a secondary requirement, even though they have become an integral part of the machining processes as this helps to reduce the temperature in the cutting zone, to transport chips, and to lubricate the tool-chip interface. Any discussion of cutting fluid requirements must include the fact that manufacturing impetus since the days of the industrial revolution is to machine parts at the highest rate of speed with maximum tool life, minimum downtime, and the fewest possible part rejects (scrap), all while satisfying accuracy and finish requirements [1].

Another environmental aspect concerns energy. In the coming years, energy will be a decisive factor both in terms of cost and competitiveness, and substantial benefits could be gained if the overall energy requirements of various machining processes are decreased. However, careful consideration must be given not only to the energy required to remove a certain amount of material but also to the energy drawn by the auxiliary equipment of a machining 
system as well as to any other aspect that may influence the technical and economical performance of the system.

\subsection{Process level considerations}

The increasing environmental consciousness in society within the last few decades can be thought of as a reflection of numerous opportunities to improve the environmental performance of manufacturing, and the elimination of the cutting fluid used in machining processes seems to have great potential. Even though cutting fluids have been seen traditionally as a solution rather than a problem, they have a variety of environmental liabilities associated with human chronic diseases and costly schemes applied for their disposal. According to German automotive industry surveys, $7 \%$ to $17 \%$ of the manufacturing cost of components is attributable to cutting fluids when associated costs of cutting fluid procurement, monitoring, maintenance, health precautions, and absenteeism are taken into account and are several times higher than the tool costs which in the same report are quoted at $2 \%$ to $4 \%$ [2].

The control of the occupational exposures and health effects and exposure assessment has been intensively studied [3-7]. Moreover, several organizations such as US Occupational Safety and Health Administration, US National Institute for Occupational Safety and Health, and US Environmental Protection Agency are very active in promoting health and safety guidance through substantial standards related to occupational exposure to metalworking fluids and aerosols.

These opportunities encouraged research into dry machining and the drastic minimization of cutting fluid use. However, the replacement of the basic functions of the cutting fluid during dry machining has proven challenging. The reduced use of cutting fluid in machining operations could result in lower tool life, increased tooling costs, and lowered productivity. For many manufacturers, this is an unacceptable compromise. Manufacturers must be convinced that for a particular application the benefits and savings from minimum quantity lubrication (MQL) and dry machining are not overshadowed by a potential for increased tool cost and lower productivity [8].

It is important to find a way of accomplishing machining processes without or with little cutting fluid and, at the same time, promoting long tool life and good workpiece quality. The most widely implemented near-dry machining method is MQL which delivers tiny amounts of lubricant mixed with compressed air directly to the point of cut. Weinert et al. [9] provided an excellent reference about dry and MQL key technologies. This keynote paper highlights the problems encountered, lists and compares different technological solutions (cutting fluid formulations, tool substrates, and coatings), and summarizes the best applica- tion area between MQL and dry machining with respect to different materials and various machining processes.

In considering environmentally friendly machining processes, the interaction between economy, ecology, and technology has to be investigated. A conflict arises between these three factors, and a good compromise based on the accurate evaluation of a vast amount of data has to be sought [10].

In the search for solutions and ways to improve machining processes, the definition of a "process unit" is a useful tool in order to develop relationships between manufacturing parameters and environmental impact. Munoz and Sheng [11] developed a manufacturing model by taking into account the material, energy, and time dimensions. Two main loss streams and their associated mass, namely the primary mass loss, which consists of chips generated in the process, and the catalytic mass losses consisting of the waste streams of the cutting fluid and the expended tools were identified.

Srinivasan et al. [12] developed a scoring scheme, Health Hazard Score (HHS), based on chemical species dose and effects along the dimensions of oral toxicity, inhaled toxicity, carcinogenicity, dermal irritation, eye irritation, flammability, and reactivity using information from databases pertinent to both the chemical industry and the occupational health and safety community. Two additional factors, the effect of waste stream on exposure route and the effect of site-specific conditions on waste containment and handling, were also included in the HHS index [13].

In the case of milling process plan assessment, some sound models and software have been developed by the laboratory of Professor Sheng at the University of California, Berkeley proposing an integration of the environmental factors in process planning. The research was conducted at the microplanning level [14] and macroplanning level, respectively [15], aiming to give the process planner the setups and the sequence of operations that decrease the total environmental burden.

Choi et al. [16] established an assessment model for manufacturing processes in terms of environmental impact for quantitative evaluation of product design. The assessment methodology was developed on the basis of the material balance of process and the relationship between different processes.

Addressing the cutting fluid as one of the main sources of environmental pollution and targeting minimization of the environmental impact related to cutting fluids, the Machine Tool-Agile Manufacturing Research Institute, developed an analytical cutting fluid evaluation tool in order to estimate the impact for flood and jet application of the cutting fluid in turning, milling and drilling processes. The Cutting Fluid Evaluation Software Testbed [17] out- 
puts quantitative information regarding the cutting fluid process performance, environmental impact, health and safety hazard scoring, and the costs for procurement as well as for treatment based on input information related to the cutting fluid type, application method, machining parameters, and site-specific factors.

The optimal selection of the cutting fluid to be used in a machining process was addressed by Tan et al. [18] who proposed a decision-making framework model considering jointly quality, cost, and environmental impact factors. The cutting fluid selection problem is also approached in [19] aiming to find the best performing cutting fluid when several different machining operations are to be performed on the same machine tool.

\subsection{System level considerations}

Process-centered efforts can be an extremely efficient means for achieving the goal of reducing environmental impact through the minimization of the amount of waste generated and by diminishing the release of hazardous substances. However, the machining processes cannot be considered as totally stand-alone processes but may be viewed as operational units within the machine tool system. Beyond the adoption of new machining strategies or changing the process parameters, opportunities for reducing the environmental impact reside also in the minimization of resource consumption, and within this category, one of the main concerns is the amount of energy consumed by the overall machining system in order to support the completion of the machining processes.

Generally, research studies focus on the cutting energy in machining systems, that is, the amount of energy required to remove a specific amount of material. However, from the point of view of green manufacturing, the energy consumption should be considered systematically for the whole machine tool system and not limited solely to the cutting energy which represents just a variable amount highly dependent on process parameters.

Challenged by the reduction of the energy consumption, the research community introduced and discussed new energy-related issues in machining. A qualitative interpretation of the energy consumption entailed by the peripheral equipment required for the preparation of the process which is not directly concerned with the cutting itself was provided in [20]. Other researchers carried out an experimental work and ascertained the importance of the energy consumption associated with non-production modes of various production machines $[21,22]$.

In agreement with the Life Cycle Assessment (LCA) policy, Narita et al. [23] developed an evaluation system of the environmental burden generated in machining in terms of global warming potential which is calculated from the electric consumption of a machine tool, coolant, lubricant oil, cutting tool status, and metal chip and converted to equivalent $\mathrm{CO}_{2}$ emissions by using appropriate emission intensity factors.

With tighter industrial regulations and environmental aspects across the globe, machine tool customers are becoming more and more interested in ecological machine alternatives. However, how well a machine tool system will be accepted as environmentally friendly depends also on the technical performances and economical impact imposed by the adoption of new technologies.

In the literature, various papers proposed decision support system models based on the analytical hierarchy process in order to solve the machine tool selection problem $[24,25]$. The selection is made mainly based on process requirements with respect to technical and economical criteria. The presence or absence of certain features such as a mist collector or fire extinguisher in consideration of safety and environmental criteria can be considered in the selection, but no attempt to quantify the use phase energy consumption of the machine tool system was made.

\section{Research aim and scope}

The decision problem in today's manufacturing industry can be summarized as one simple question: How is it possible to achieve economic growth by taking advantage of the latest technologies while protecting the environment?

From the published work, it can be inferred that, due to the diverse nature of metal removal, there is no sovereign remedy that applies to all aspects of the machining processes. Making a choice between alternative machining strategies is not simple, especially when more than one criterion must be taken into account. If simultaneous improvements were feasible, then we could machine a part with zero cost, perfect quality, and no environmental impact which is totally unrealistic. Actually, the improvement of one factor is not always possible without the worsening of another one when considering a complex system and an elaborate set of criteria.

Motivated by these aspects, the scope of this research work is to develop tools and enhance knowledge related to the minimization of the environmental impact and to the reduction of the resource consumption in machining processes. The minimization or complete reduction of the cutting fluid in machining processes as well as the decrease of the energy requirements in machining could be a powerful indicator for sustainable manufacturing. Generally, there are two main ways to approach the contribution of machining systems and processes to the environmental load, either an absolute approach that makes a direct link to environmental effects such as ozone depletion, greenhouse 
effect, smog, etc...or a relative approach assuming that a reduction in the use of materials and energy yields an environmental benefit.

The framework introduced in this paper for the evaluation of the use phase of machine tool systems does not calculate explicitly the environmental impact but enables the evaluation of environmental factors in accordance with the aforementioned relative approach. Moreover, economical and technical aspects are also considered allowing the decision maker to be in a better position to make sustainable decisions.

Since the simultaneous improvement of all the criteria to be taken into account is almost impossible to achieve, the aim of the proposed method is to find a compromise solution by using the analytic hierarchy process (AHP) which provides a comprehensive and rational environment for structuring the decision problem at both process and system level.

\section{General information on the evaluation methodology}

\subsection{What is an MTS?}

The machine tool system (MTS) can be thought of as an evolution of a traditional machine tool endowed with numerical control and able to perform different mechanical work types on different faces of the same workpiece. Basically we can distinguish two main functions that must be satisfied simultaneously by the system in order to get the maximum technical, economical, and environmental benefits from the machining processes, namely to remove material and to support/control the material removal.

The former function is mostly concerned with the motions necessary to get the workpiece cut and the structural configuration of the machine tool, implicitly deciding the movement capabilities and the degrees of freedom of the system, whereas the latter function is assured by the control and peripheral equipment that gathers together all the auxiliary systems necessary to sustain and ensure free-of-trouble serviceability of the machine tool during its use.

\subsection{Why consider the evaluation of the use phase?}

Nowadays, a life cycle thinking approach is more and more preferred in the design of every product. LCA methods identify the material, energy, and waste flows associated with a product over its entire life cycle so that the environmental impacts can be determined. LCA aims to give a measure of environmental effects of the life cycle of a product as an average over time and location which means that LCA is not an appropriate tool to assess the environmental impact at a given location and moment, as is the case when the MTS is in use.

The environmental impact in the use phase of active products such as an MTS which generates other products can become easily more important than that from other life cycle phases. Use processes are performed by executing functions of the MTS. Most of the environmental impact in machining stems from energy use, but this does not result just from deliberated functions. It rather results from the behavior of each MTS subsystem responsible for a specific function. Unlike manufacturing or end of life phases which are under the direct control of corporations, the use of the system passes to the customer, which makes the environmental impact much more difficult to evaluate. Our interest is focused on the actual use phase when a clear delimitation between the machined product-specific and machined product non-specific use processes should be made.

\subsection{How is the evaluation process structured?}

Broadly speaking, the methodology envisages the evaluation of two main factors: the cutting area with its local effects and the activities of the peripheral equipment as long as the MTS is in use. Accordingly, the evaluation is conducted at two levels (see Fig. 1).

Level 1, purely experimentally based, considers the local cutting environment along with the "actors" that are directly involved in the cutting area, their relationships, and the phenomena that occur from their interactions. Level 2, based on the specifications of various MTS components and an energy consumption predictor module, characterizes the activities performed by the MTS during the use phase.

Nowadays, considering sustainable development stakes, no decisions in manufacturing could be made without considering environmental issues. In a complex system such as MTS, the environmentally friendly decisions could be taken at different levels since this complexity might offer the opportunity to initiate different actions intended to improve the overall performance of the system.

The elimination of hazardous substances from the machining processes is one of the actions that has led to the implementations of new machining strategies. One of these strategies, to be implemented at the process level, envisages reduction or even complete elimination of the cutting fluid from the machining processes. Another decision would be to increase the efficiency of the energy usage of the entire system. In order to produce a final part, the MTS has to process the part through several steps, with each step requiring a certain amount of energy.

Although significant information related to the performance of machine tools, machining processes, cutting tools, and materials already exists, there is no unified methodol- 
Fig. 1 Machine tool system overview

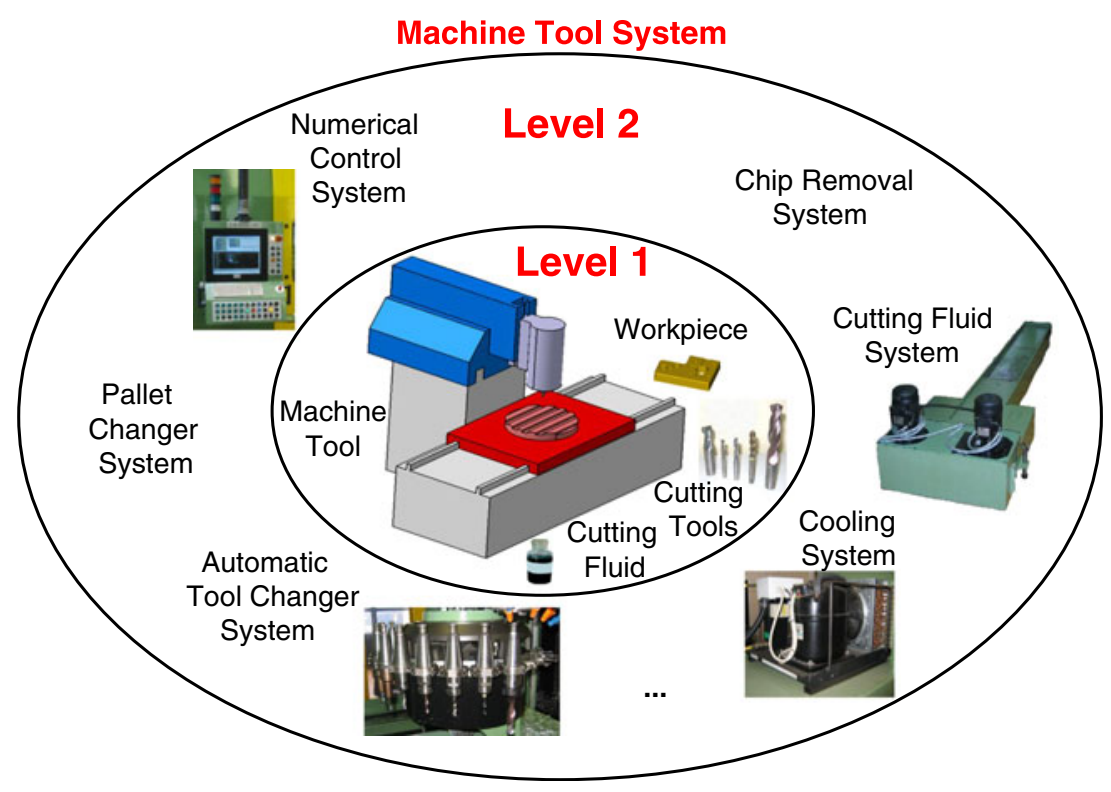

ogy to combine all this information to give a meaningful justification for the choice of a certain machining strategy in the manufacturing of a specific part. In other words, the methodology targets providing fundamental knowledge regarding the relationship between the effects of choosing a machining strategy defined by the corresponding cutting tool, cutting parameters, tool path, type and application method of the cutting fluid for a given workpiece material, and the impact generated by the entire machining system as a result of its employment.

Decision making can be regarded as a process of selection among several alternatives. An alternative refers to the choice between several possibilities all intended to accomplish a specific goal. At the process level, the focus is on various alternatives for cooling/lubrication of the cutting area whereas at the second level, the various possibilities to fulfill the auxiliary functions which generally provide assistance for the cutting activities are addressed.

\subsection{What criteria for the comparison of the alternatives?}

Reliable terms of comparison are needed to confront the pros and cons of every alternative. A set of consistent criteria is proposed as the basis of comparison. Each level counts on a number of distinct criteria, but both levels group the criteria under the same three categories: economical, technical, and environmental. The decision regarding the selection of the criteria for both levels has been supported by several arguments formulated on behalf of each alternative under the corresponding category. The criteria modeling scheme is open for rearrangements as the arguments provided reflect merely the vision of the authors on the classification of each criterion under a specific category.

\subsubsection{Machining economics}

Two criteria which present economical importance for the first level are the tool life and the machining time. The primary functions of the cutting fluid are to cool and to lubricate the cutting area. If no fluid is used, these functions seem to be taken over by the tool through coatings. On the one hand, the coating technology not only made dry machining possible but also raised the cost of the tool. On the other hand, the high cost of the tool may not be completely justified under wet or MQL conditions, which can meet the machining requirements with uncoated, less expensive tools due to the presence of the cutting fluid in the process. Thus, in order to clarify how the cutting tool and the process are affected by the alteration of the cooling/ lubrication conditions, experiments focused on the comparative measurement of the tool life are of major interest.

As is to be expected, an MTS should perform several operations for the purpose of reaching the final shape of a part. Each operation necessitates a certain amount of time to be completed. Any reduction of the time spent in machining a specific feature can be considered as an economical improvement of the process.

The shift of the economical analysis from the first level to the evaluation of the entire system brings into discussion two criteria: the cost, this time involving actions and components of the whole system, and the productivity. If the cost analysis is to be extended to the entire system, the focus is on aspects such as the initial capital expenditure, the additional costs resulting from the purchase of fixturing or any other auxiliary device, the cost for fixed and variable maintenance, the operator hourly wage, etc...

Nowadays, the continuous demand for improvement in production of quality products through an efficient, effec- 
tive use of the machining resources makes productivity one of the most important criteria to be considered in the evaluation of the MTS. To be more specific, MTS productivity is a measure relating a quantity or quality of machined products to the resources required to produce them and consequently depends on several specifications of the system such as spindle power, maximum cutting speed and feed, tool change time, pallet change time, rapid traverse rate, etc...

\subsubsection{Technical aspects}

Certainly, a thorough evaluation of a machining process when the question that arises is either to continue employing cutting fluids or to eliminate them completely must involve specific technical criteria. In consequence, cutting forces and the workpiece quality are proposed as criteria for the first level, whereas precision and flexibility/adaptability cover the technical aspects of the second level.

The cutting forces are widely recognized as a performance estimator of the machining processes. These forces, being the result of extreme conditions at the tool-workpiece interface, determine the spindle power requirements and bearings loads, cause deflection of the part, tool, or machine structure, and transfer energy to the machining system which may result in excessive cutting temperatures or unstable vibrations.

The quality of surface finish is considered as being a technical criterion since it is commonly specified along with linear and geometric dimensions and often determines how well a part performs. Each machining process ordinarily produces surface finishes in a certain range, and generally, to improve surface finish quality requirements implies additional processes and incurring increased machining costs. It is important that no better finish than really needed be specified for a surface. In the context of the developed methodology, the surface roughness is considered, as it is perhaps the most widely spread quality characteristic of a surface generated through a machining process.

Nowadays, machining systems with a high-quality precision design are compulsory in order to satisfy market needs. Hence, perhaps the most important factors affecting the quality of a machine and implicitly the quality of the final machined products are the accuracy, repeatability, and resolution of its components and the manner in which they are combined. These factors are critical because they affect every one of the parts that will be manufactured using the MTS. Any component that has a movement capability at the same time has an important contribution for the precision reached by the system.

The presence of various auxiliary systems made possible improved machine utilization, part scheduling efficiency, part setup, and handling time. In principle, an MTS with a higher degree of flexibility can handle a variety of similar or dissimilar part designs and can enable fast setups for new part designs to be introduced quickly into production. Flexibility depends on the number of axes, number of pallets, number of tools, the table size, the maximum travel value of each axis, etc...

\subsubsection{Environmental concerns}

In the past, machining decisions were usually made based only on improving the productivity level or quality of a product, so the process or material that has the shortest process time will eventually be chosen disregarding other aspects such as energy consumed or the impact generated by different waste streams issued during machining processes.

Dry and MQL machining offer great promise for reducing or eliminating various environmental issues associated with wet machining, mostly related to cutting fluid management along its use. Unlike a perishable cutting tool which is being continuously used and can wear off in a very short period of time, the cutting fluid can be used for a much longer period of time. The cost of the cutting fluid management is shared by all the parts machined during the useful life of the fluid. A different situation arises in the case of MQL since the application of the fluid is performed without any recovery. For the sake of consistency, the "cutting fluid consumption" criterion is exclusively considered as the rate the fluid is delivered in the cutting area and all the other aspects are neglected.

In the absence of the cutting fluid that could trap an important amount of particles generated, dry and MQL machining are inherently more likely to release metal dust which may pose both inhalation and explosion risks. Potential trade-offs need to be evaluated, in order to characterize the air quality of machining processes under dry, MQL, and wet conditions in terms of the size of particles generated, usually expressed in micrometers.

Energy, and particularly electricity, whose use has increased significantly since the start of the industrial revolution, is now an essential part of society, and the general perception about its consumption is mostly reflected in the price that has to be paid for its use. As a matter of fact, the biggest problem that should enhance the user's consciousness about energy consumption is the current way of producing energy that leads to enormous and possibly irreversible environmental damage. The energy criterion is shared by both levels and any reduction of its use pertaining either to the removal of a specific amount of material or to the requirements of the peripheral equipment can be considered as an environmental benefit. 
Modern MTS should be configured to ensure a high level of protection for operator, environment, and the system itself. The identification of the system components along with their specifications that contribute to the preservation of a safer and cleaner working environment is an issue of high interest for the assessment of the control performance of unavoidable emissions released during machining.

\section{Methodology overview}

The adoption of new machining strategies is an important issue for any machine tool system. In real machining decision conditions, more than one criterion is present and the problem becomes a multi-criteria decision-making one. The methodology proposed involves the AHP technique applied for structuring decision hierarchies at both process and system levels.

\subsection{A multi-criteria decision-making approach}

Nowadays, gaining the maximum benefits during the use phase of an MTS is one of the main goals of the manufacturing industry, and therefore, an integrated approach that jointly addresses the assessment of different machining strategies and MTS aptitudes to accomplish potential tasks during its lifetime can lead to a harmonized and consistent decision.

In this article, we propose a multiple criteria decisionmaking approach for solving the evaluation of the MTS use phase when there are conflicting criteria. A multiple criteria decision-making approach seeks to find one or several satisfactory alternatives among a set of possible alternatives. Alternatives are judged by several criteria, which are conflicting in almost all real-life decision problems. By interaction with the decision maker, preference information is captured and used to evaluate alternatives.

The AHP method, firstly developed by Saaty [26], divides a complicated system under study into a hierarchical system of elements. The whole task of the decision maker(s) is made simpler by constructing a hierarchy and developing a mathematical model that generates the priority values for different criteria and subcriteria as involved in the decision-making process.

The AHP helps to compare pairwise the criteria at a particular level of the hierarchy to find out which criteria the decision maker wants to set with the highest priority. While comparing those criteria qualitatively, some corresponding scale values are assigned to them. Table 1 shows the 1 to 9 scale with the corresponding interpretations.

These criteria comparison ratings $a_{i j}$ are generally used to develop a near consistent criteria comparison matrix. A consistent matrix is a positive reciprocal matrix $n \times n$, whose elements satisfy the relation $a_{i j} \times a_{j k}=a_{i k}$ for $i, j, k=$ $1, \ldots, n$.

The elements $a_{i j}, i, j=1, \ldots, n$ are the estimation of the dominance of the criterion $i$ over $j$ and satisfy the condition $a_{i j}>0, a_{i j}=\frac{1}{a_{i j}}$. Thus, the pairwise comparison matrices are positive reciprocal matrices.

Having a pairwise comparison matrix, the next step is to compute the priority vector which is the normalized eigenvector of the matrix. First of all, the sum of the ratings in each column is computed and then each element of the matrix is divided by the sum of its column. This is followed by the calculation of the normalized principal eigenvector which can be obtained by averaging the normalized relative weights across the rows. The normalized principal eigenvector is also called the priority vector and shows the relative weights among the criteria compared. Since it is normalized, the sum of all elements in the priority vector is 1 .

Apart from the computation of the relative weights of the criteria, the consistency of the pairwise comparison should be checked. To do that, the principal eigenvalue is needed, which is obtained from the summation of products between each element of the eigenvector and the sum of columns of the reciprocal matrix. The eigenvalue is used to assess the strength of the consistency ratio of the comparative matrix and determine whether to accept the information.

Saaty [26] proved that for a consistent reciprocal matrix the largest eigenvalue is equal to the size of the comparison matrix that is $\lambda_{\max }=n$. In general a positive reciprocal matrix is inconsistent and $\lambda_{\max } \geq n$. Furthermore, since small variations in $a_{i j}$ cause variations in $\lambda_{\max }$, a measure of consistency called the consistency index (CI) was defined through the formula $\mathrm{CI}=\frac{\left(\lambda_{\max }-n\right)}{n-1}$. The calculated consistency index reflects the decision maker's judgment and should be compared with that of a randomly generated reciprocal matrix on the scale $1-9$ which is called the random index (RI). The random index values are depicted in Table 2.

The ratio of CI to the average RI for the same order matrix is called the consistency ratio (CR). If we are perfectly consistent, then the consistency measure will be equal to $n$, and therefore, the CI will become zero and so will the $\mathrm{CR}$. As a rule of thumb, the CR should be about 0.1 or less with regard to an acceptable decision. If the $\mathrm{CR}$ is small, the estimates are accepted; otherwise, any higher value warrants a re-examination of the judgment.

The AHP hierarchical structure of the first level is depicted in Fig. 2. Unlike the process-level evaluation (first level), which applies a rating assignment procedure only for the criteria level, the system-level (second level) analysis needs more computation to achieve the preference index since the judgment is based on ordering the basis of comparison between alternatives in three levels: category, 
Table 1 Possible criteria rating scale values and corresponding interpretations

\begin{tabular}{ll}
\hline Scale value & Interpretation \\
\hline 1 & Equally preferred \\
3 & Moderately preferred \\
5 & Strongly preferred \\
7 & Very strongly preferred \\
9 & Extremely preferred \\
$2,4,6,8$ & Halfway between the integers on either side \\
Reciprocals of above & In comparing criteria $i$ and $j$, if $i$ is 3 compared to $j$, then $j$ is $1 / 3$ compared to $i$ \\
\hline
\end{tabular}

criteria, and subcriteria. The AHP hierarchical structure of the second level, defined using the approach of Cimren et al. [25], is depicted in Fig. 3 .

A machining strategy can be capable of high technical performances but may be more harmful to the environment. Conversely, a strategy can offer important ecological gains but might be economically expensive and technically unsatisfactory. For instance, the use of cutting fluids in machining process guarantees a good surface finish, but in return, the cutting fluid use entails high costs and an important environmental burden related to its management. Dry machining could be a viable solution, since no fluid at all is used in the process, but if the same tool and machining parameters are kept, the tool will get worn faster engendering a surface finish that will hardly meet the specifications. Therefore, the decision maker has to make a compromise between several criteria in order to select a machining strategy for particular conditions.

Following the same logic, the compromise case also holds true for the second level. For instance, productivity, precision, and the energy consumed during the use of an MTS are matters of utmost importance. While the MTS precision is defined by its architecture and joints as well as the manufacturing and assembly quality of its components, productivity and energy consumption are also strongly dependent on the machining strategies. However, the trend is to get the most from the MTS in terms of productivity, experiencing elevated levels of precision with an amount of energy consumed that must be reduced as much as possible.

\subsection{Process level evaluation}

The impact assessment of a certain machining strategy is based on a set of experiments performed for a machining feature. The objective is to conduct simple studies at the local feature level for alternative strategies. An alternative strategy is defined by changing one parameter in the part process plan, a change that is supposed to bring an improvement of one or several aspects of the process performance. For each strategy created, a set of criteria that must fall under the economical, technical, and ecological categories is monitored. Generally, an MTS performs several machining processes in order to achieve the final shape of a part. An overview of the elements characterizing the evaluation at the first level is depicted in Fig. 4.

The entire amount of data needed for the level 1 analysis is stored in the "Results" database whose structure is presented as an entity relationship diagram.

The first level approach is a feature-process-based approach. That is to say that for each feature belonging to a part, the "Results" database is requested to supply existing data related to machining that feature. At this initial stage, a given part is divided into its component features, and measurements made for each of these are sought among the tests existing in the database. This process is repeated for all the features contained in a part, and then, by an aggregation of the multi-criteria analysis results achieved for each feature, a final score for the overall part will be obtained that coincides with the accomplishment of the level 1 analysis.

Searching for the most appropriate strategy employed to machine a part is a process of selection among a number of machining alternatives under a pre-defined set of criteria. The total number of measurements made for each test is grouped by the entity "MeasuredCriteria". The 1:1 cardinality existing between the entities "Test" and "MeasuredCriteria" allows easy retrieval of the values of measurements done for a specific experiment. The list of criteria is opened for new entries by a simple operation of adding new attributes in the "MeasuredCriteria" entity.

Table 2 Average random index values

\begin{tabular}{llllllllll}
\hline Order of matrix $(n)$ & 2 & 3 & 4 & 5 & 6 & 7 & 8 & 9 & 10 \\
\hline RI & 0 & 0.58 & 0.9 & 1.12 & 1.24 & 1.32 & 1.41 & 1.45 & 1.51 \\
\hline
\end{tabular}


Fig. 2 Hierarchical structure of the first level (process level)

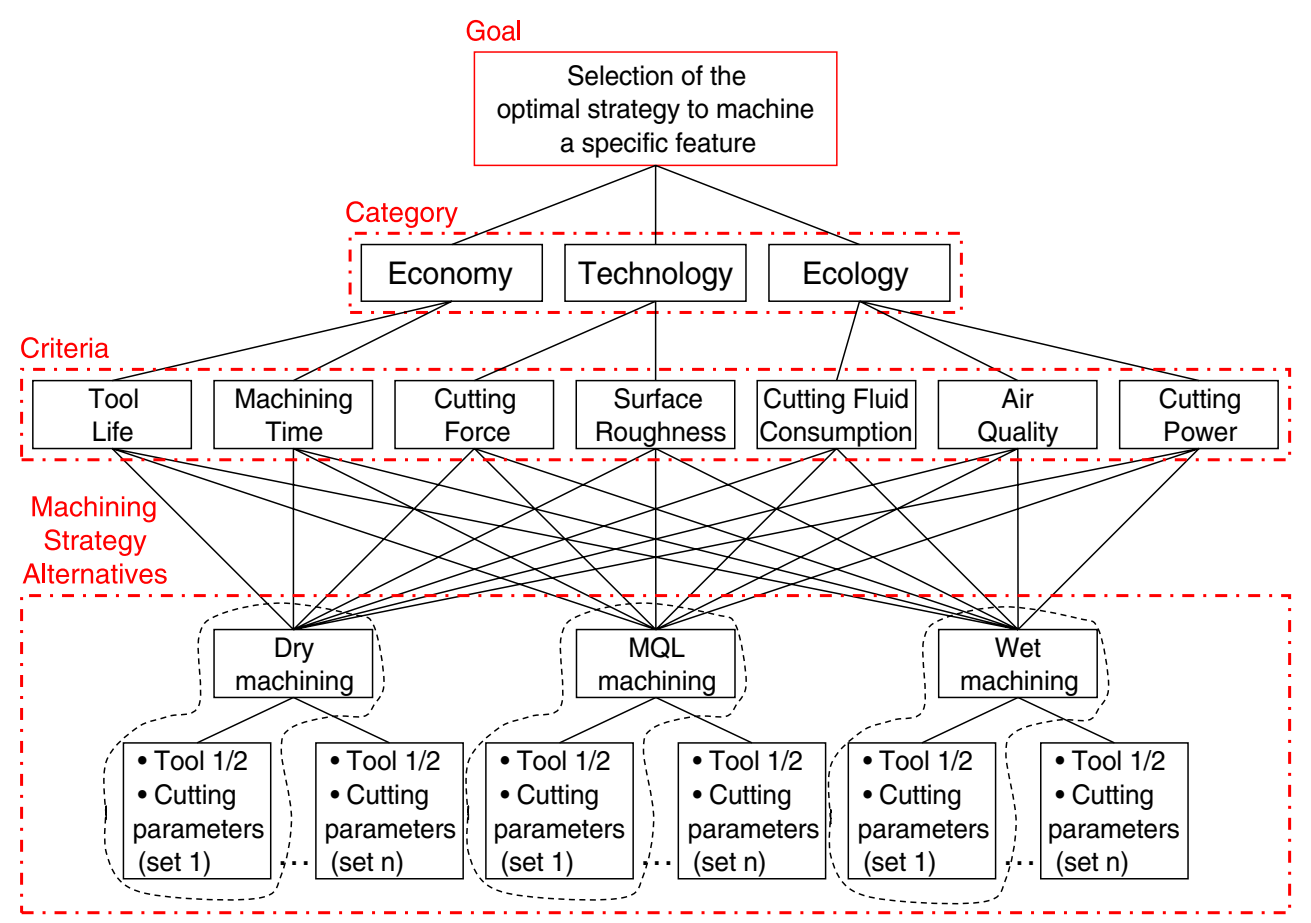

\subsection{System level evaluation}

The MTS assessment from the point of view of its capability to fulfill competitively different machining requirements during the use phase presents a high interest. In this respect, a realistic result cannot be pointed out only with an assessment of the cutting area occurrences. Therefore, in order to accomplish the evaluation, a second level that gives special attention to the activities of the peripheral equipment of the system is brought into the discussion.

The output of the machining system mostly depends on the performances of the constituent subsystems. Imperative to the efficiency of the machine tool is the way the MTS capabilities are matched with the work to be done. The part
Fig. 3 Hierarchical structure of the second level (system level)

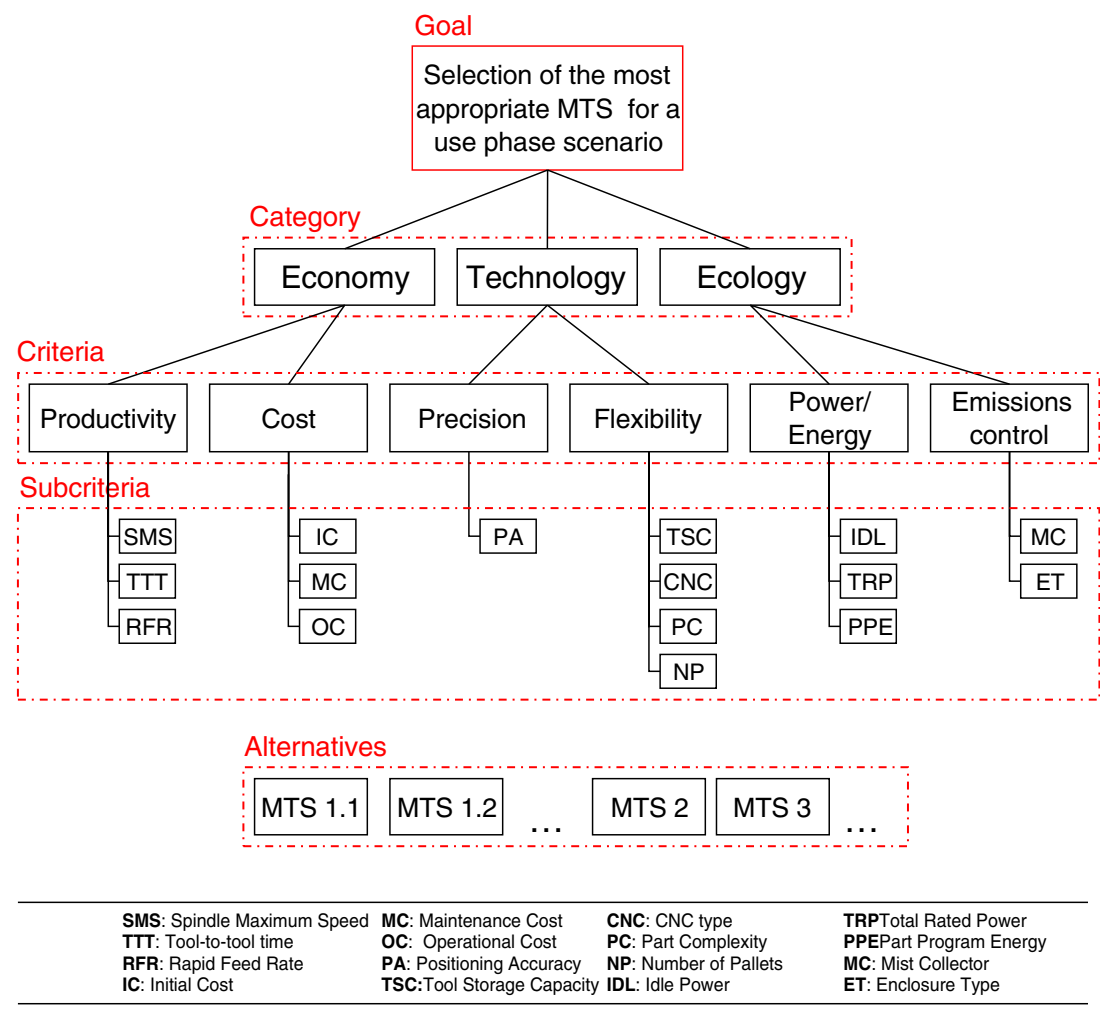



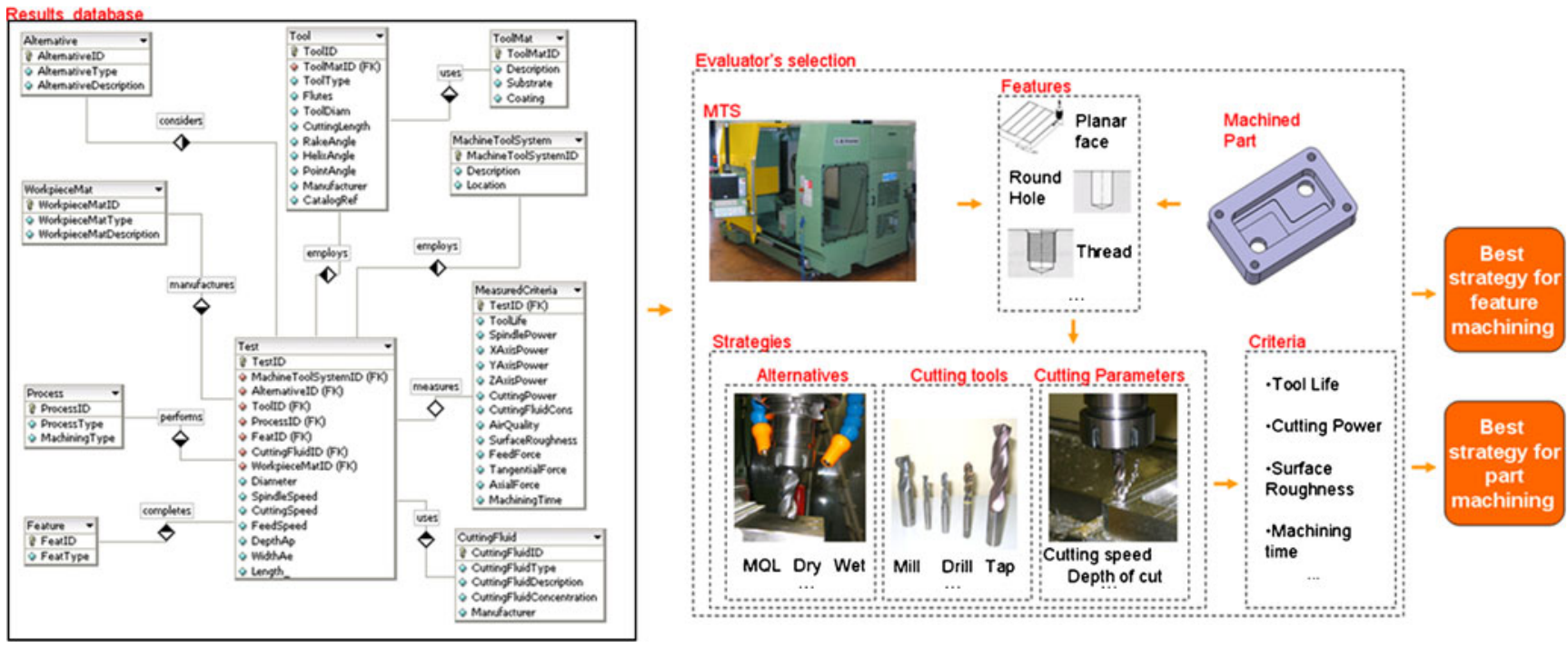

Fig. 4 Overview of the level 1 analysis

range and the production strategy chosen should allow using the machine tool in the most efficient manner. A large reserve of resources incompletely exploited is very likely to result in a useless rise in cost.

The system is subjected to a trade-off between the same three main aspects as employed for the process evaluation: economy, technology, and ecology, as can be seen in Fig. 3. A set of criteria and subcriteria is proposed under each category. The decision situation is characterized by both quantitative and qualitative subcriteria. Basically, the quantitative values assigned to each subcriterion represent technical and economical specifications of the main subsystems of the MTS contributing to the overall performance of the system while in use. The values corresponding to the three subcriteria under the criterion "Power/Energy" are also quantitatively expressed.

The idle power stands for the power drawn by a specific machine as long as it is turned on without performing any machining task. This value can be derived from measurements. The second subcriterion, the total rated power, represents the sum of the rated power values of all the electric components of the machine tool system. The part program energy resides on an analytical model that estimates the power requirements of the spindle and feed axes with respect to a given part program. By integrating these power requirements with respect to the machining time, the cutting energy can be obtained. In addition, the power requirements of the auxiliary equipment involved in the accomplishment of the part program, as derived from measurements, are also considered in order to determine the overall energy consumption of the MTS for the machining of a part. The "PartProgramEnergy" entity of the "Resource" database was designed for the storage of this information, under the "TotalEnergy" attribute.
The criterion "Emissions Control" is broken down into two qualitative subcriteria reflecting the system's capability for handling the emissions from the processes performed.

Information about the machine tool component performances forms one of the primary foundations necessary to enable manufacturing of any part to specifications and on schedule. The "Resource" database stores this information according to the entity relationship diagram presented in Fig. 5.

Unlike the "Results" database that groups explicitly all the alternatives that refer to a specific machining strategy under the same entity entitled "Alternative", within the "Resource" database, the alternatives are found as records in every entity that describes one of the main subsystems of the MTS. This means that each record of a certain entity constitutes an alternative of a subsystem by describing physical components and their specifications designed to accomplish one of the MTS functions. One MTS alternative is outlined by gathering together corresponding alternatives for each subsystem.

The "Resource" database configuration makes suitable easy storage and retrieval of distinct MTS alternatives described in terms of specifications of system components. Furthermore, the representation of a new alternative starting from an MTS that underwent either a modification in the specifications of a certain system or has been retrofitted with a new system could also be easily achieved. These can be considered as derivations of a base MTS alternative (i.e., alternatives labeled as MTS 1.1, MTS 1.2, etc... in Fig. 3).

\section{Implementation}

The proposed methodology is implemented in a software tool (Global Reasoning for Eco-Evaluation of Machining) 


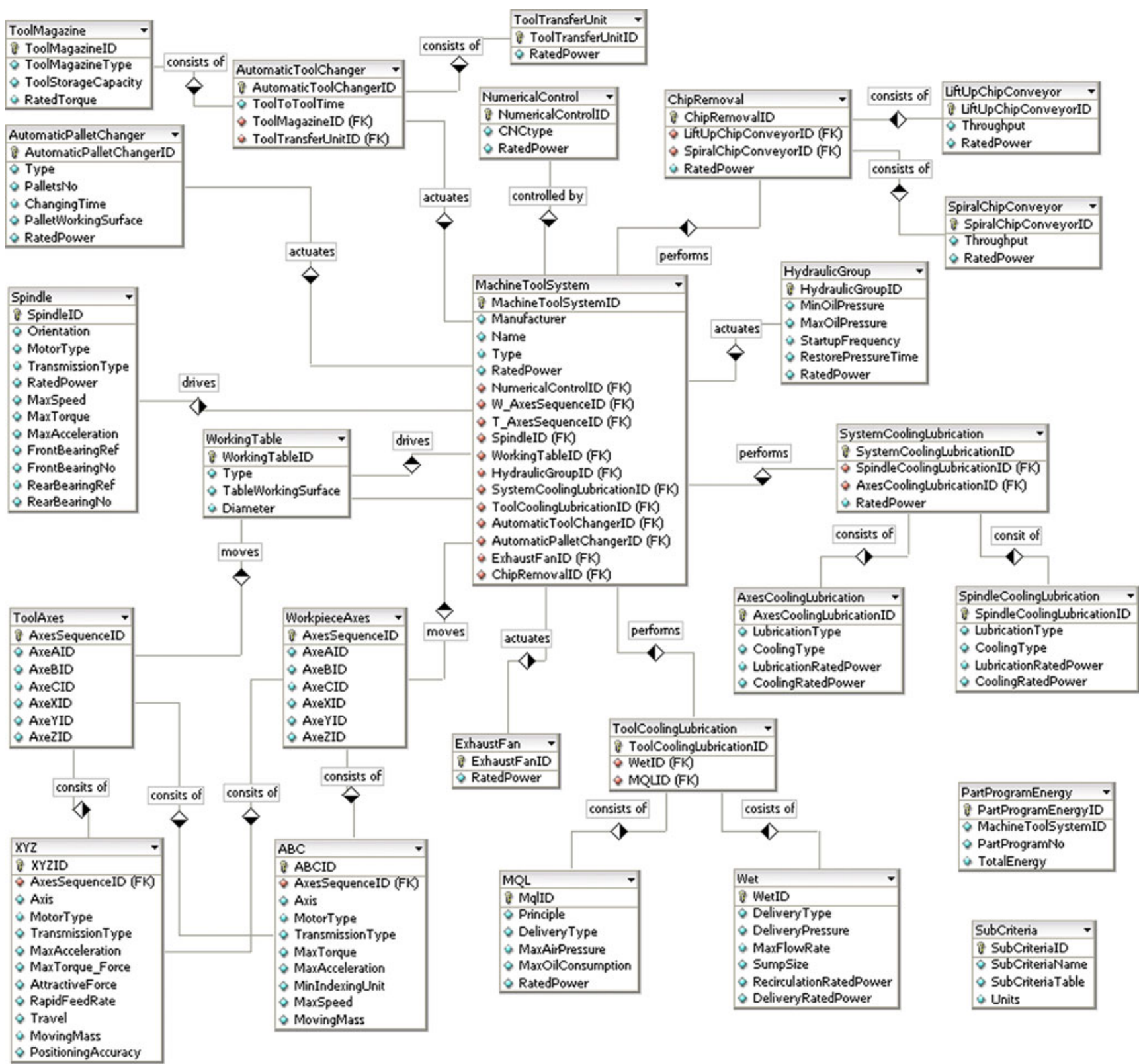

Fig. 5 Resource database-entity relationship diagram

based on Visual Basic for Applications. Microsoft Access was chosen as a database management system, allowing relatively quick development because all the tables, queries, forms, and reports can be stored in the database.

\subsection{Level 1}

The "Results" database, designed for easy data access, gathers data concerning tests performed by several MTSs. Input is taken through a user-friendly interface that helps the user both enter all necessary data and get the results of the analysis. The entire analysis is structured as a six-step procedure, with each step grouping specific actions.

- Step 1: Experimental table (retrieve machining data)

Initially, in the first step called "ExperimentalTable", the MTS that is to be analyzed has to be selected from a list containing all the names/types of the MTS introduced in the database. Simultaneously, the connection with the "Results" database is established and will be kept all the way through the completion of the level 1 analysis.

As soon as the connection has been established, the user can start the retrieval of the experiments in the database. 
After deciding on the MTS and on the workpiece material, the selection of the features, alternatives, and criteria will follow. Then the cutting tool specifications and cutting parameters will complete the database query. Once all the aforementioned elements are specified, the experimental table can be produced and the first step is complete.

- Step 2: Criteria consistency (determine criteria relative weights)

The second step deals with the relative importance of the measured criteria specified by the user. The software determines the consistency of the decision maker's judgment and the weights to be assigned to the criteria based on which the evaluation is carried out following the AHP methodology.

- Step 3: Elementary analysis (evaluate an individual feature over all criteria)

In the "Elementary Analysis" phase, which is the third step, the evaluation is done separately for each feature. This allows the user to identify contributions and options for setting parameters in the process plan of a part. In order to do so, a normalization procedure must be performed since the criteria values have different measurement units and their direct aggregation is not possible.

The normalization is performed by searching the minimum and maximum for each row in the experimental table, that is to say that for each criterion, the minimum and maximum experimentally determined feature evaluation values are sought among the values corresponding to all the strategies tested for a specific feature. Afterward, according to the direction of preference (minimization or maximization) setup for each criterion, two formulas apply: $v^{\prime}=(v-\min ) /(\max -\min )$ for minimization and $v^{\prime}=$ $(\max -v) /(\max -\min )$ for maximization, respectively, where $v$ is the value to be normalized.

The normalized values are multiplied by the criteria' coefficient of importance assigned as a result of the application of the AHP. The results generated by this multiplication are summed across all criteria in order to gather together under one normalized value the contribution of each criterion involved in the evaluation of each alternative considered. For the same feature, the sums obtained are compared with each other with respect to the same alternative. Consequently, a ranking of the tests with respect to each alternative can be established, with the test that got the lowest sum being rated the best. Each test brings together a cutting tool, a set of cutting parameters, and a tool path in a unique combination.

- Step 4: Global analysis (aggregate ranking of alternatives for machining a part)

The fourth step is the global analysis which relies on an aggregation of the results over all features belonging to a part from the previous step. The global analysis step will provide two outcomes: a ranking of all the alternatives considered and the best option for machining a part with respect to a set of criteria. The former outcome is based on the ranking of the total score of each alternative obtained by summing the scores of the best performing tests under the same alternative for every feature included in the evaluation. This offers a clear picture of the burden of each alternative, facilitating the user's judgment in choosing the alternative that best fits his or her needs.

The latter outcome proposes the best way to machine the part disregarding the clustering of the performed tests around one alternative. In this case, the best performing test is sought among all alternatives for each feature and then the corresponding values are summed.

- Steps 5 and 6: Graphical representation and reporting

The last two steps, namely the "Graphical Representation" and "Reporting", summarize through graphical tools and a text file the results obtained along the entire evaluation.

\subsection{Level 2}

The second level of the software addresses the performance of the entire machining system with respect to several criteria. The main steps to be followed for the completion of this analysis are described below:

\section{- Step 1: MTS selection}

The first step consists in the selection of two MTSs to be compared. If level 1 was completed, then the name of the MTS previously selected is transferred directly to the MTS selection input window. Although the software is developed under the consideration of a simultaneous comparison between several MTSs, the multiple MTS assessment part has not been implemented. Instead, the comparison is made between two systems defined as the MTS to be evaluated and the MTS reference, respectively.

- Step 2: Criteria consistency (determine criteria relative weights)

Similarly to the first level evaluation, the AHP is used, which in this case is meant to evaluate what type of MTS is most appropriate for a machining scenario with respect to the hierarchical structure described in Fig. 3.

\section{- Step 3: Power requirements}

In this step, the energy requirements of the spindle and feed axes of a machine tool system are estimated based on 


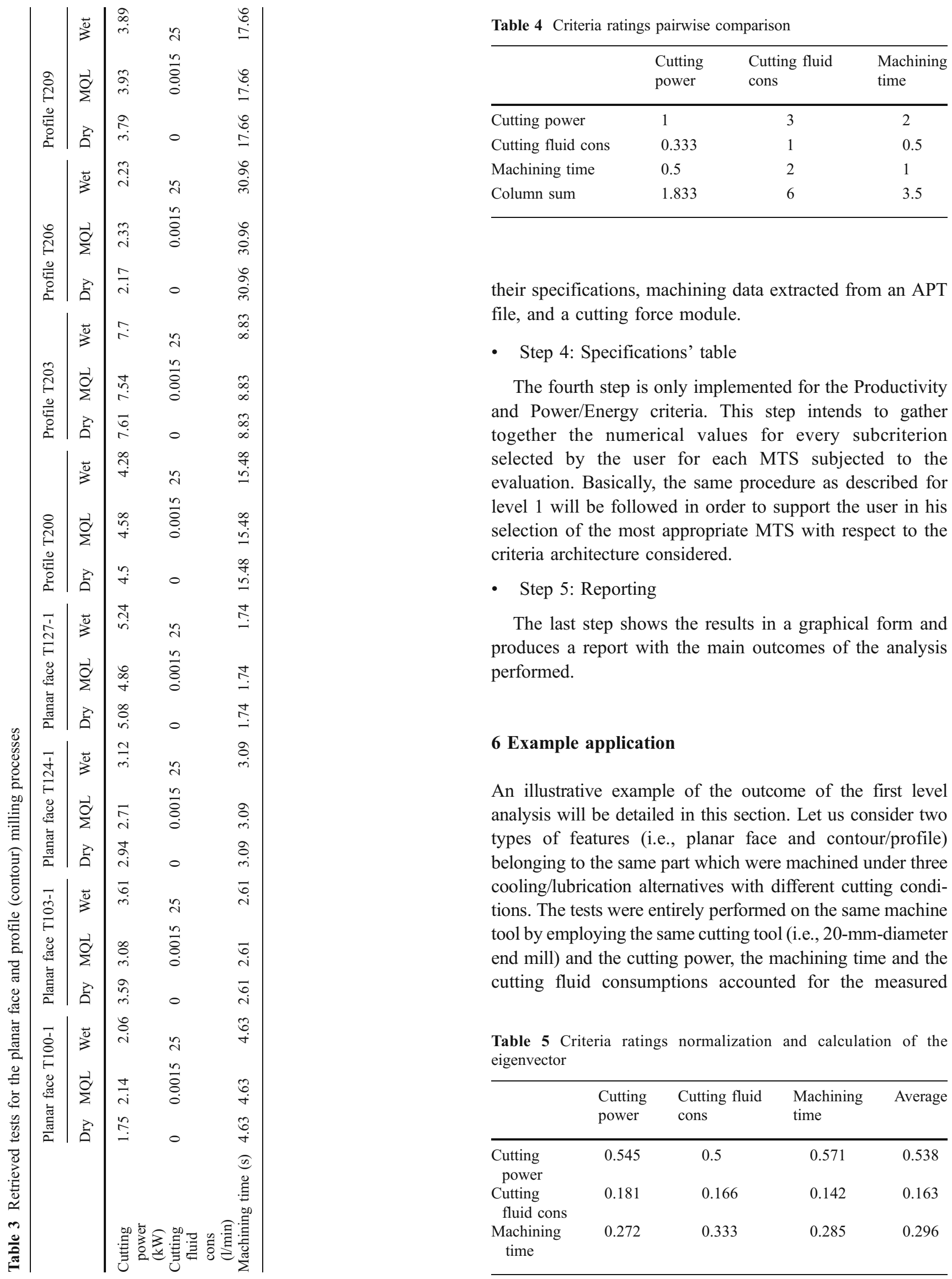


Table 6 Summary of the judgment consistency indices

\begin{tabular}{llccc}
\hline & Eigen value & Consistency index & Random consistency index & Consistency ratio \\
\hline Cutting power & $1.619 / 0.538=3.009$ & 0.005 & 0.58 & 0.0086 \\
Cutting fluid cons & $0.490 / 0.163=3.006$ & & \\
Machining time & $0.891 / 0.296=3.01$ & & \\
\hline
\end{tabular}

criteria. All these information are collected from the user through a GUI and are used to query the "Results" database in order to identify the appropriate machining tests.

All the data pertaining to the tests compliant with the user query are tabulated individually for each feature in the "ExperimentalTable" spreadsheet as depicted in Table 3.

The retrieved tests are numbered with respect to the cutting conditions specified by the user during the database querying. The retrieval of the data satisfying the user requirements is followed by the criteria weighting. This involves pairwise comparison between the three selected criteria. Let us assume a pairwise comparison matrix as depicted in Table 4.

According to the ratings given in the table above, the preference for the criterion "CuttingPower" is three times more important than the "CuttingFluidCons" and two times more important than the "MachiningTime", respectively. Since we have three criteria to be compared, one more comparison is needed to complete the reciprocal matrix. In this respect, the preference for criterion "MachiningTime" is as much as twice as important that the "CuttingFluidCons". It can be observed that the diagonal elements of the matrix are always one, and the lower triangular matrix is filled with the reciprocal values of the ratings assigned in the upper triangular matrix.

The next step is to normalize the matrix, that is, to calculate the sum of the ratings in each column and then divide each rating by the corresponding sum. The normalized principal eigenvector can be obtained by averaging across the rows (see Table 5).

The principal eigenvalue with respect to the first criterion is calculated by multiplying the average rating for each criterion with the scores in the first row one-ata-time followed by the sum of these products and the division of this sum by the average rating for the first criterion. This calculation, which involves a matrix multiplication, can be handled with the "MMULT" function available in Excel:

$$
\left(\begin{array}{ccc}
1 & 3 & 2 \\
0.333 & 1 & 0.5 \\
0.5 & 2 & 1
\end{array}\right)\left(\begin{array}{l}
0.538 \\
0.163 \\
0.296
\end{array}\right)=\left(\begin{array}{l}
1.619 \\
0.490 \\
0.891
\end{array}\right)
$$

The consistency index can be calculated as a function of the maximum eigenvalue estimated for the three criteria and the size of the matrix. Table 6 shows the main indices needed to ascertain the judgment consistency with respect to the criteria ratings pairwise comparison.

As soon as the judgment consistency has been validated (consistency ratio $\leq 0.1$ ), we can calculate the weight corresponding to each criterion by simply dividing the sum of each row by the total sum of the rows (see Table 7).

Since each criterion has a different measurement unit, a direct aggregation of the performances of each machining strategy alternative with respect to all criteria considered is not reasonable. Therefore, a normalization of the values stored in the "ExperimentalTable" spreadsheet is compulsory. In this example, we consider that all the three criteria are to be minimized which results in the employment of the minimization formula for the normalization process (i.e., normalized value $=($ criterion value - minimum row value $) /$ (maximum row value-minimum row value)). The normalized criteria values and normalized sums are stored in the corresponding tables in the "ElementaryAnalysis" spreadsheet as shown in Tables 8 and 9.

This step (elementary analysis) is completed after the identification of the best performing machining strategy for each feature. Due to the minimization preference for the values recorded across all tests for the three criteria, the best performing test is indicated by the lowest normalized sum for each feature. As a result, the cutting parameters corresponding to test T124-1 in the presence of the MQL cooling/lubrication are preferred for the machining of the planar face feature whereas for the profile feature, dry machining and the cutting conditions corresponding to test T209 are better suited with respect to the criteria considered.

In the following step, the user will be provided with the ranking of the cooling/lubrication alternatives employed to machine one complete part considered as a sum of features. Basically, the global analysis relies on the individual performance of every feature previously analyzed and aggregates these performance values under

Table 7 Criteria weighting

\begin{tabular}{llcl}
\hline & Row sum & Total row sum & Weight \\
\hline Cutting power & 6 & 11.333 & 0.5294 \\
Cutting fluid cons & 1.833 & & 0.1618 \\
Machining time & 3.5 & & 0.3088 \\
\hline
\end{tabular}




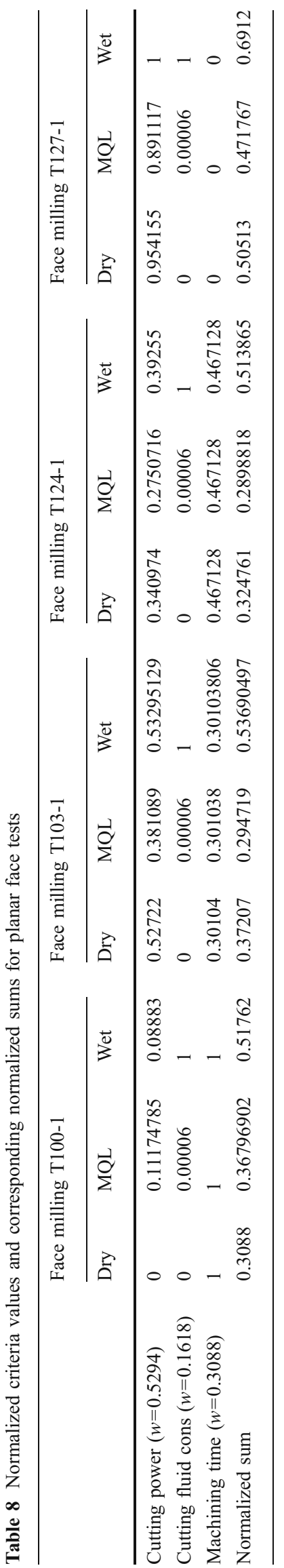

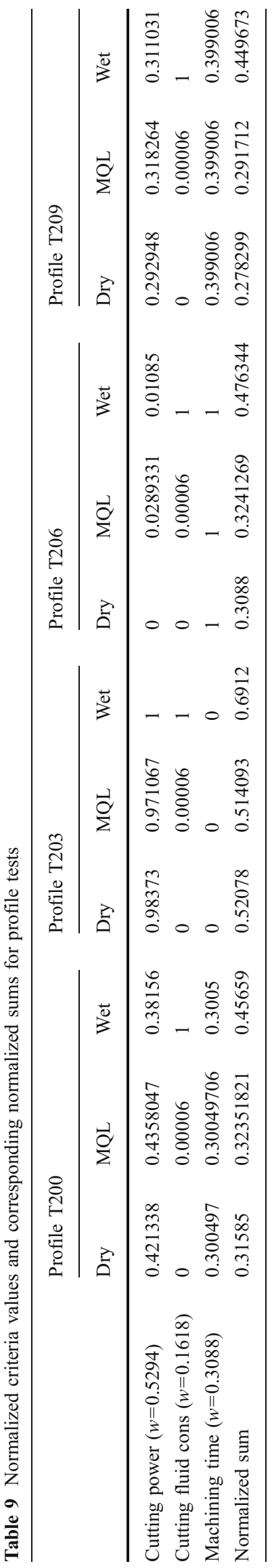


Fig. 6 Overview of the global analysis results

\begin{tabular}{|c|c|c|}
\hline Planar face T124-1 & Mql & 0.28988177 \\
\hline ProfileT209 & Dry & 0.27829945 \\
\hline total score & \multicolumn{2}{|c|}{0.568} \\
\hline \multicolumn{3}{|l|}{ best mgl } \\
\hline Planar face T124-1 & Mal & 0.28988177 \\
\hline Profile T209 & MqI & 0.29171169 \\
\hline total score & \multicolumn{2}{|c|}{0.58} \\
\hline \multicolumn{3}{|l|}{ best dry } \\
\hline Planar face T100-1 & Dry & 0.3088 \\
\hline ProfileT209 & Dry & 0.27829945 \\
\hline total score & \multicolumn{2}{|c|}{0.587} \\
\hline \multicolumn{3}{|l|}{ best wet } \\
\hline Planar face T124-1 & Wet & 0.51386518 \\
\hline ProfileT209 & \multirow{2}{*}{\multicolumn{2}{|c|}{\begin{tabular}{l|l} 
Wet & $\mathbf{0 . 4 4 9 6 7 2 6 9}$ \\
\end{tabular}}} \\
\hline total score & & \\
\hline
\end{tabular}

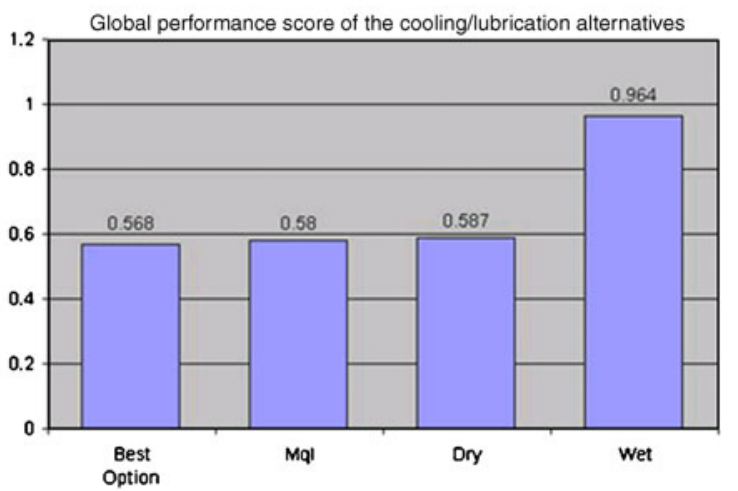

one global score. The software provides also the best option to machine a part, which gathers together the best performing tests for each feature regardless of the cooling/lubrication method used (see Fig. 6).

In the previous figure, we notice that the smaller normalized values for each feature were obtained with respect to different cooling/lubrication alternatives. Generally, this situation is to be avoided in practice since the benefits of using one cooling/lubrication alternative can be overshadowed by the inconveniences yielded by the use of one of its counterparts. In this respect, it would be more preferable to machine all the features of one part with no fluid at all in order to avoid the contamination of the machine and chips as well as extra cleaning operations of the part. For our example, the dry machining of the part is the third option but its score came very close to the first two ranked options. The analysis performed points out the wet machining as the worst scenario with respect to the criteria selected. This is mainly due to the "Cutting Fluid Cons" criterion whose values were largely higher than the values assigned for the other two alternatives which were very close to zero (MQL machining) or even zero (dry machining). This aspect is illustrated in Fig. 7 which shows the contribution of each criterion in the performance of each cooling/lubrication alternative for each feature considered.

The data retrieval from the "Resource" database, the criteria rating, the weight assignment, the normalization procedure, and the searching process for the best performing alternative are followed in the same manner as described for level 1. A noticeable change consists in the increasing complexity of the criteria structure, and in the way, the energy criterion is dealt with.

\section{Discussion}

It is worthwhile mentioning that the software implemented to demonstrate the methodology concept is able to handle different machining strategies applied to machine features not only in the context of a complete part but also when
Fig. 7 Criteria contribution in the performance score of the best performing facing and contouring tests

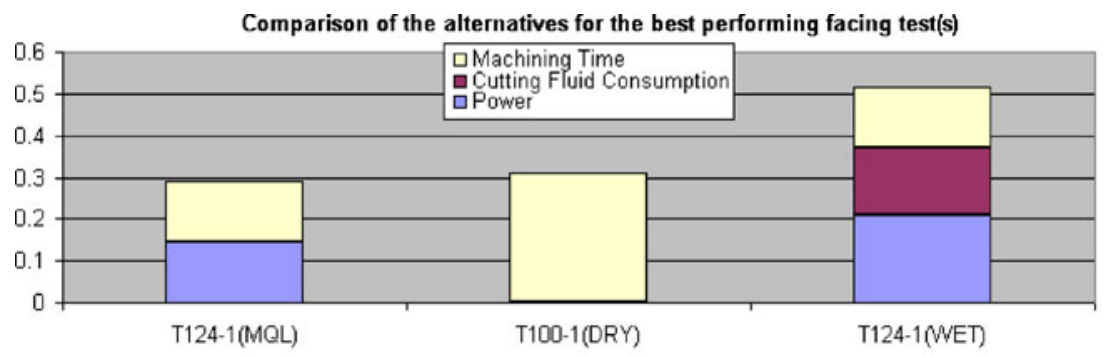

Comparison of the alternatives for the best performing profile test(s)

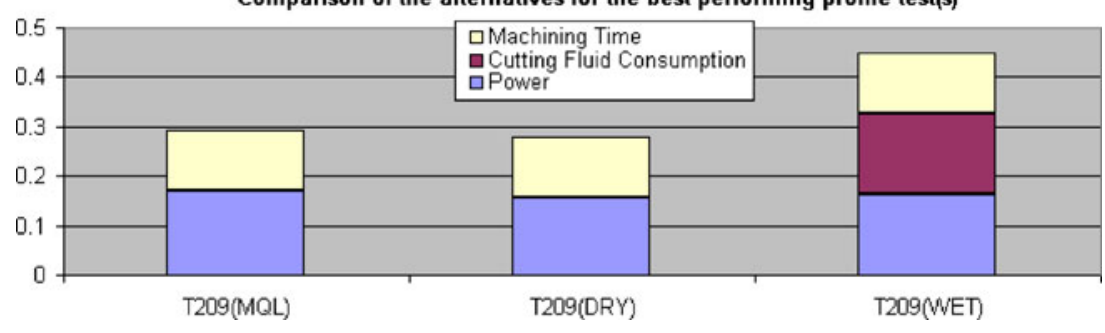


they are considered as individual features. In other words, there is no need to have features exclusively belonging to a part; just having several tests performed for the same feature with respect to several strategies and stored in the "Results" database is the only condition that must be fulfilled to make the evaluation possible.

Even though the analysis could be performed without considering the full set of criteria, for the purpose of an accurate analysis, it is preferable to have a complete database able to supply values for as many criteria as possible. At least one criterion from each category must be involved in the assessment if an insightful outcome is expected. For instance, productivity, precision, and energy consumption of an MTS can be considered together due to the strong interaction that exists between them. An increase in productivity can be associated with an increase in the automation level of the system so that the idle times could be drastically reduced but not without side effects since a higher level of automation would necessitate a greater amount of electricity from the power grid causing a higher environmental load. The quality of the final part is also reflected by the precision characteristics of the MTS components. If the quality prescribed for a part cannot be achieved by an MTS, additional processes or even additional MTSs are needed to meet the requirements, which means not only a reduction of the productivity rate but also the expenditure of a higher amount of energy.

At the first level, the analysis provides the best compromise solution either for the machining of individual features or for the machining of various prismatic parts through an aggregation of the performances of the component features. This outcome serves as a guideline for setting cutting parameters and tool cooling/lubrication alternatives to improve the performance of specific machining processes with respect to a consistent set of measured criteria characterizing the interaction between the tool, workpiece, and the cutting fluid. In addition, the contribution of each criterion in the total performance of an alternative is readily available allowing the decision maker to identify opportunities for further improvements.

\section{Conclusions}

In this paper, a unified methodology developed for the evaluation of the use phase of the MTS with respect to economical, technical, and environmental criteria was presented. Since the simultaneous improvement of all the criteria to be taken into account is almost impossible to reach, the method aims to find a compromise solution by using the AHP which provides a comprehensive and rational environment for structuring the decision problem at both process and system level. A consistent set of criteria and an entity relationship diagram have been introduced for each level. The reduction/ elimination of the hazardous substances (i.e., cutting fluid) and the reduction of the resource consumption (i.e., energy) are the main environmental considerations addressed by the two aforementioned levels.

The process level relies on measurement data stored in a database especially developed for this purpose and on the priority values assigned to a set of criteria in order to carry out a feature-/process-based performance assessment. Technical and economical specifications of the machine together with quantitative power data which can be derived either from rated power specifications of the various MTS components, analytical models, or from measurements are employed for the analysis carried out at the system level.

Acknowledgments The authors gratefully acknowledge the partial financial support from the EU FP6 project NEXT (Next Generation Production Systems) under Grant No. NMP2-CT-2005-011815.

\section{References}

1. Byers JP (2006) Metalworking fluids, 2nd edn. Taylor and Francis, New York

2. Klocke F, Eisenblatter G (1997) Dry cutting. CIRP Ann 46 (2):519-526

3. El Baradie MA (1996) Cutting fluids: part II. Recycling and clean machining. J Mater Process Technol 56(1-4):798-806

4. Sheng PS, Oberwalleney S (1997) Life-cycle planning of cutting fluids: a review. J Manuf Sci Eng 119b(4):791-800

5. Sokovic M, Mijanovic K (2001) Ecological aspects of the cutting fluids and its influence on quantifiable parameters of the cutting process. J Mater Process Technol 109(1-2):181-189

6. Gauthier SL (2003) Metalworking fluids: oil mist and beyond. Appl Occup Environ Hyg 18(11):818-824

7. Sheehan MJ, Hands D (2007) Metalworking fluid mist-strategies to reduce exposure: a comparison of new and old transmission case transfer lines. Appl Occup Environ Hyg 4(4):288-300

8. McClure TF, Gugger MD (2002) Microlubrication in metal machining operations. Lubr Eng 58:15-21

9. Weinert K, Inasaki I, Sutherland JW, Wakabayashi T (2004) Dry machining and minimum quantity lubrication. CIRP Ann 53 (2):511-537

10. Byrne G, Scholta E (1993) Environmentally clean machining processes - a strategic approach. ClRP Ann 42(1):471-474

11. Munoz AA, Sheng P (1995) An analytical approach for determining the environmental impact of machining processes. $\mathrm{J}$ Mater Process Technol 53:736-758

12. Srinivasan M, Wu T, Sheng P (1995) Development of a scoring index for evaluation of environmental factors in machining processes: part1-formulation. Trans NAMRI 23:115-121

13. Gune P, Sheng P (1995) Influence of site specific factors in environmentally conscious manufacturing. Proc ASME IMECE Symp Life-Cycle Eng, San Francisco, CA

14. Srinivasan M, Sheng P (1999) Feature based process planning for environmentally conscious machining-part I: microplanning. Rob Comput Integr Manuf 15(3):257-270

15. Srinivasan M, Sheng P (1999) Feature based process planning for environmentally conscious machining-part II: macroplanning. Rob Comput Integr Manuf 15(3):271-281 
16. Choi ACK, Kaebernick H, Lai WH (1997) Manufacturing processes for environmental impact assessment. J Mater Process Technol 70:231-238

17. Sutherland JW, Cao T, Daniel CM, Yue Y, Zheng Y, Sheng P, Bauer D, Srinivasan M, DeVor RE, Kapoor SG, Skerlos SJ (1997) CFEST: an internet based cutting fluid evaluation software testbed. Trans NAMRI 25:243-248

18. Tan XC, Liu F, Cao HJ, Zhang H (2002) A decision-making framework model of cutting fluids selection for green manufacturing and a case study. J Mater Process Technol 129(1):467-470

19. Axinte D, Axinte M, Tannock J (2003) A multicriteria model for cutting fluid evaluation. Proc IMechE B J Eng Manuf 217 (10):1341-1353

20. Akbari J, Oyamada K, Saito Y (2001) LCA of Machine Tools with regards to their secondary effects on quality of machined parts. Proceedings EcoDesign 2001: second international symposium on environmentally conscious design and inverse manufacturing, Tokyo
21. Dahmus JB, Gutowski TG (2004) An environmental analysis of machining. Proceedings of IMECE, Anaheim, California, USA

22. Devoldere T, Dewulf W, Deprez W, Willems B, Duflou J (2007) Improvement potential for energy consumption in discrete part production machines. Proceedings of 14th CIRP international conference on life cycle engineering, Waseda, Tokyo, Japan, 1113 June, pp 311-316

23. Narita H, Kawamura H, Norihisa T, Chen LY, Fujimoto H, Hasebe $\mathrm{T}$ (2004) Prediction system of environmental burden for machining operation. Proceedings of 2004 Japan-U.S.A. symposium on flexible automation, Denver, USA, July 2004

24. Arslan MC, Catay B, Budak E (2004) A decision support system or machine tool selection. J Manuf Technol Manage 15(1):101-109

25. Cimren E, Budak E, Catay B (2007) Development of a machine tool selection system using analytic hierarchy process. J Adv Manuf Technol 35:363-376

26. Saaty TL (1990) How to make a decision: the analytic hierarchy process. Eur J Oper Res 48:9-26 